\title{
Evaluation of procedures for typing of group B Streptococcus: A retrospective study
}

\author{
Hans-Christian Slotved ${ }^{\text {Corresp., }}{ }^{1}$, Steen Hoffmann ${ }^{1}$ \\ ${ }^{1}$ Neisseria and Streptococcus Reference Laboratory, Department of Microbiology and Infection Control, Statens Serum Institut, Copenhagen, Denmark \\ Corresponding Author: Hans-Christian Slotved \\ Email address: hcs@ssi.dk
}

Background. This study evaluates two procedures for typing of Streptococcus agalactiae (group B streptococci; GBS) isolates, using retrospective typing data from the period 2010 to 2014 with a commercial latex agglutination test (latex test) and the Lancefield precipitation test (LP test). Furthermore, the genotype distribution of phenotypically non-typable (NT) GBS isolates is presented. We also raise the awareness, that the difference in typing results obtained by phenotypical methods and genotype based methods may have implications on vaccine surveillance in case a GBS vaccine is introduced.

Methods. 616 clinical GBS isolates from 2010-2014 were tested with both a latex test and the LP test. Among these, 66 isolates were genotyped by PCR, including 41 isolates that were phenotypically NT.

Results. The latex test provided a serotype for $83.8 \%$ of the isolates (95\%, C.I. $80.7-86.6)$ compared to $87.5 \%$ (95\%, C.I. 84.6 - 90.0) obtained by the LP method. The two assays provided identical capsular identification for all sero-typeable isolates (excluding NT isolates). The PCR assay provided a genotype designation to the 41 isolates defined as phenotypically NT isolates.

Discussion. We found that the latex test showed a slightly lower identification percentage than the LP test. Our recommendation is to use the latex agglutination as the routine primary assay for GBS surveillance, and then use the more labour intensive precipitation test on the NT isolates to increase the serotyping rate. A genotype could be assigned to all the phenotypically NT isolates, however, as a consequence genotyping will overestimate the coverage from possible future capsular polysaccharide based GBS vaccines. 
1

2

3

4 Hans-Christian Slotved ${ }^{1 *}$, Steen Hoffmann ${ }^{1}$

$5 \quad{ }^{1}$ Neisseria and Streptococcus Reference Laboratory, Department of Microbiology and Infection

6 Control, Statens Serum Institut, Copenhagen, Denmark.

\section{Evaluation of procedures for typing of group B Streptococcus: A retrospective study}

12 Corresponding author:

13 H-C Slotved*

Running title: Typing of group B Streptococcus isolates

\author{
s.
}

Hans-Christian Slotved

Department of Microbiology and Infection Control, Statens Serum Institut.

Artillerivej 5, DK-2300 Copenhagen, Denmark

Phone: +45 32688422, E-mail: hcs@ssi.dk.

8

9

0

\title{
1
}

2

23 


\section{ABSTRACT}

Background. This study evaluates two procedures for typing of Streptococcus agalactiae (group B streptococci; GBS) isolates, using retrospective typing data from the period 2010 to 2014 with a commercial latex agglutination test (latex test) and the Lancefield precipitation test (LP test). Furthermore, the genotype distribution of phenotypically non-typable (NT) GBS isolates is presented. We also raise the awareness, that the difference in typing results obtained by phenotypical methods and genotype based methods may have implications on vaccine surveillance in case a GBS vaccine is introduced.

Methods. 616 clinical GBS isolates from 2010-2014 were tested with both a latex test and the LP test. Among these, 66 isolates were genotyped by PCR, including 41 isolates that were phenotypically NT.

Results. The latex test provided a serotype for $83.8 \%$ of the isolates $(95 \%$, C.I. $80.7-86.6)$ compared to $87.5 \%(95 \%$, C.I. $84.6-90.0)$ obtained by the LP method. The two assays provided identical capsular identification for all sero-typeable isolates (excluding NT isolates). The PCR assay provided a genotype designation to the 41 isolates defined as phenotypically NT isolates.

Discussion. We found that the latex test showed a slightly lower identification percentage than the LP test. Our recommendation is to use the latex agglutination as the routine primary assay for GBS surveillance, and then use the more labour intensive precipitation test on the NT isolates to increase the serotyping rate.

A genotype could be assigned to all the phenotypically NT isolates, however, as a consequence genotyping will overestimate the coverage from possible future capsular polysaccharide based GBS vaccines. 


\section{Introduction}

Streptococcus agalactiae (group B streptococcus, GBS) is a well-known pathogen primarily causing infections in newborns and the elderly (Brigtsen et al., 2015, Ballard et al., 2016). The disease in neonates is generally described as occurring in two different varieties (Bulkowstein et al., 2016). Early-onset disease (EOD) occurs in the neonate during the first six days of life, while late-onset disease (LOD) occurs later than the seventh days of life and can develop up to three months of age (Le Doare \& Heath, 2013, Vinnemeier et al., 2015). Possible clinical manifestations of GBS infection in neonates are sepis, meningitis and pneumonia (Schrag et al., 2013). Among adults, GBS may also be associated with invasive infections, particularly in elderly persons with underlying medical conditions (Le Doare \& Heath, 2013). Since introduction of screening programmes for pregnant women (Ballard et al., 2016) in some parts of the developed world, particularly early onset GBS infections in neonates has been reduced, and for many years the GBS disease incidence has been low (Heath, 2016). In contrast, early onset GBS infection is still a major problem in the developing world and presumably an underestimated problem (Heath, 2016). Also in recent years, the developed world has seen an increasing interest in incidence of invasive GBS infections, in particular among the elderly (Sheppard et al., 2016). Surveillance and identification of GBS in humans are therefore increasingly essential (Ballard et al., 2016, Sheppard et al., 2016).

The GBS are currently divided into ten serotypes based on type specific capsular antigens and are designated as Ia, Ib, II, III, IV, V, VI, VII, VIII, and IX (Slotved et al., 2007, Le Doare \& Heath, 2013). For decades, the precipitation test also known as the Lancefield precipitation test (LP test) has been considered the standard method for GBS serotype determination (Slotved, Sauer \& Konradsen, 2002). However, the method is time-consuming and therefore not suited for 
71 typing large number of isolates (Slotved et al., 2003). At present, GBS isolates are in general

72 serotyped by the phenotypical method latex agglutination test (latex test) (Afshar et al., 2011),

73 for which several kits are commercially available. Increasingly simpler and affordable molecular

74 techniques for genotyping of GBS isolates, predominantly based on PCR assays, have been

75 introduced and are now commonly used (Brigtsen et al., 2015, Sheppard et al., 2016).

76 In recent years, all GBS isolates received at the Statens Serum Institut (SSI) have been typed

77 using both a LP test and a latex test (Slotved, Sauer \& Konradsen, 2002, Lambertsen et al.,

78 2010). Furthermore, some of the GBS NT isolates have been tested for genotype using the PCR

79 assay described by Imperi et al. (2010) and Poyart et al. (2007).

By using all our GBS typing data from the period 2010 - 2014, we evaluated the phenotypical

81 typing procedure for GBS isolates, based on the comparison of the commercial latex test and the

LP test. We will furthermore show the genotype distribution of phenotypically NT GBS isolates.

\section{Methods}

This is a retrospective study based on typing data obtained in the period from 2010 to 2014 at the national Neisseria and Streptococcus Reference Laboratory (NSR), SSI.

The Danish hospitals are serviced by regional departments of clinical microbiology, all of which are public. On a voluntary basis, they submit isolates of beta-hemolytic streptococci to the NSR for national surveillance (Lambertsen et al., 2010).

$\underline{\text { Isolates }}$ 
92 The study was based on 616 isolates received at the NSR laboratory (SSI) in the period of 2010

93 to 2014. The majority of the isolates were from bloodstream infections and each isolate

94 represented one patient case.

95 The LP test is considered the reference method at the NSR laboratory (SSI) (Slotved, Sauer \&

96 Konradsen, 2002, Lambertsen et al., 2010).

97

$\underline{\text { Identification of GBS isolates }}$

The GBS isolates were identified as described by Lambertsen et al. (2010). Briefly, the submitted strains were examined for their characteristic beta-hemolytic colonies on $5 \%$ horse blood agar plates (SSIDiagnostica, Denmark) followed by serogrouping with group B latex (Oxoid A/S, Greve, Denmark) as recommended by the manufacturer. Isolates were stored at $80^{\circ} \mathrm{C}$ in nutrient beef broth containing $10 \%$ glycerol (SSI Diagnostica).

$\underline{\text { Serotyping of the GBS isolates }}$

All isolates were tested both with the LP test and latex test (SSI, Diagnostica) (Lambertsen et al., 107 2010).

$\underline{\text { Lancefield precipitation test (LP test) }}$

110 The precipitation test was performed as described by Slotved, Sauer \& Konradsen (2002).

111 Briefly, a resuspended centrifuged overnight broth culture was boiled and treated with $0.2 \mathrm{~N}$

112 hydrochloric acid $(0.2 \mathrm{~N} \mathrm{HCl})$ to extract the capsular antigen. A LP test was performed, by 113 mixing the extract with serotype specific GBS antisera (Ia - IX) (SSIDiagnostica, Denmark). If 
114 no reaction occurred then an extract with $0.1 \mathrm{~N} \mathrm{HCl}$ was made and tested. Non-serotypeable

115 isolates were designated NT.

116 See also http://www.ssidiagnostica.dk/Produkter/Antisera/Streptokok-antisera/Streptokok-

117 antisera-til-praecipitation (Accessed 10-02-2017) for a video description of the LP test.

118

$119 \underline{\text { Latex agglutination test (latex test) (ImmuLex TM, SSIDiagnostica, Denmark) }}$

120 The latex test was performed with the Streptococcus latex test ImmuLex ${ }^{\mathrm{TM}}$ (SSIDiagnostica,

121 Denmark). Briefly, isolates were cultured for 24 hours in Todd-Hewitt broth. Ten microlitres

122 from this culture was mixed with $10 \mu 1$ specific antisera corresponding to one of each of

123

124

125

126

127

128

129

130

131

132

133

134

135

136

serotypes Ia, Ib, and II-IX specific to capsular polysaccharide antigen latex test suspension, and agglutination was read after 5 to 10 seconds (Slotved et al., 2003).

\section{$\underline{\text { PCR test }}$}

The multiplex PCR assay and primers (TAG Copenhagen) used in this study were described by Imperi et al. (2010) and Poyart et al. (2007). Briefly, $0.5 \mathrm{ml}$ Chelex solutions were prepared of each isolate. The multiplex PCR for the genes was performed using a $20 \mu 1$ PCR mix of $10 \mu 1$ HotstarTaq Mastermix (Qiagen, Hamburg, Germany). The following PCR program used was: 15 min at $95{ }^{\circ} \mathrm{C}, 35$ cycles of $15 \mathrm{sec}$ at $95^{\circ} \mathrm{C}, 50 \mathrm{sec}$ at $55^{\circ} \mathrm{C}, 60 \mathrm{sec}$ at $72{ }^{\circ} \mathrm{C}$, finalized with $10 \mathrm{~min}$ at $72{ }^{\circ} \mathrm{C}$. The presence and quality of expected PCR fragments were tested by gel-electrophoresis on $2 \%$ E-gels (Invitrogen).

In the years 2010 and 2011 we chose to evaluate the PCR on a majority of the phenotypically NT isolates. 
137

138

139

140

141

142

143

144

145

146

147

148

149

150

151

152

153

154

155

156

157

158

159

\section{Results}

Serotyping by the LP test $(0.1 \mathrm{~N} \mathrm{HCl}$ and $0.2 \mathrm{~N} \mathrm{HCl})($ table 1$)$.

Of the 616 GBS isolates tested with $0.2 \mathrm{~N} \mathrm{HCl}$, it was possible to serotype 530 isolates $(86.0 \%$, $(95 \%$, C.I. $83.0-88.6))$. The 86 isolates that did not show a serotype reaction were tested using

$0.1 \mathrm{~N} \mathrm{HCl}$, thereby identifying further nine isolates, resulting in a total identification of 539 isolates $(87.5 \%,(95 \%$, C.I. $84.6-90.0))$. The remaining 77 isolates $(12.5 \%$, $(95 \%$, C.I. $10.0-$ 15.4)) were classified as NT isolates. It was predominantly serotype III (3/185 isolates) and VIII isolates $(3 / 15)$ which were identified with $0.1 \mathrm{~N} \mathrm{HCl}$. One isolate of each of serotype Ia (1/113 isolates), serotype V (1/77 isolates), and serotype XI (1/5 isolates) were identified using $0.1 \mathrm{~N}$ $\mathrm{HCl}$.

\section{A comparison of Latex test with the LP test (table 1).}

With the latex test it was possible to serotype 516 isolates $(83.8 \%,(95 \%$, C.I. $80.7-86.6))$, while 100 isolates $(16.2 \%,(95 \%$, C.I. $13.4-19.4))$ could not be identified, due to either multiple reactions (cross-reactions) or no reactions. The latex test provided results identical to those obtained by the LP test, except for 11 isolates that were NT by the LP test. Six of these 11 isolates were serotype V. Among 100 isolates that were NT with the latex test, 34 were serotyped with the LP test (table 1).

\section{Molecular typing by PCR}

66 of the 616 isolates were tested for their genotype. Of these isolates 25 could be assigned a serotype, while 41 isolates were considered serotype NT (tables 2 and 3). The 41 NT isolates included nearly all NT isolates for 2010 (24 of total 30) and 2011 (15 of total 16) (table 2). The 
160 genotyping of the 41 isolates designated as NT by the LP test, showed a high predominance of

161

162

163

164

165

166

167

168

169

170

171

172

173

174

175

176

177

178

179

180

181

182

serotype V (15 isolates) followed by serotype III (9 isolates) (table 2$)$. Nearly all phenotypically

NT isolates could be genotyped (table 2).

When comparing the genotype and serotype by LP test of the 25 identified isolates, a difference

was noted for two isolates. Both isolates were genotype II, while they were serotype III with

$0.2 \mathrm{~N} \mathrm{HCl}$ (table 3 ). Two isolates identified as genotype V were identified as serotype VI and VII with the LP test, while they were NT with the LP test (table 4).

The combined vaccine relevant serotypes (serotype Ia, Ib and III) represented 49.7\% (95\%, C.I. $42.0-57.4)$ of phenotyped isolates in 2010 and $56.6 \%(95 \%$, C.I. $47.8-65.1)$ in 2011 . If those phenotype NT isolates, where a genotype of one of the three vaccine serotypes was established, were added to the serotype distribution, then the vaccine relevant serotypes (serotype Ia, Ib and III) represented 56.6\% (95\%, C.I. $48.9-64.1)$ in 2010 and $61.0 \%(95 \%$, C.I. $52.3-69.2)$ in 2011.

\section{Discussion}

In general, laboratories use Latex tests, and to a minor extent LP test for GBS serotyping (Sheppard et al., 2016, Afshar et al., 2011). In this study, we found the latex test able to serotype $83.8 \%(95 \%$, C.I. 80.7 - 86.6), while the LP test was able to serotype $87.5 \%$ (95\%, C.I. $84.6-$ 90.0) of the isolates (table 1). Both assays provided serotype identification to some isolates that were non-typeable with the other method, although to a varying degree (table 1). We did not find any conflicting test results using the two assays except for the NT isolates. A serotyping identification rate between $80 \%$ and $90 \%$ is common (Brigtsen et al., 2015), and even lower serotyping rates has been observed (Slotved et al., 2003, Yao et al., 2013). Because the latex test 
183 is much easier to perform than the LP test, there is no question on which phenotypical method to 184 use for routine serotyping (Brigtsen et al., 2015; Sheppard et al., 2016). At the NSR laboratory 185 (SSI) we have chosen the following procedure for phenotypical serotyping (figure 1): we start 186 with the latex test, if this method provide serotype identification, then the identification is accepted, and no further serotype testing is performed. If the latex test shows either crossreactions or non-typeability, then we proceed to testing the isolate using the LP test, by first testing for $0.2 \mathrm{~N} \mathrm{HCl}$ and then if necessary proceed to $0.1 \mathrm{~N} \mathrm{HCl}$. If the LP test provides a specific serotype, then this is accepted, or else the isolate is defined as non-typeable. Using this procedure provide a serotyping percentage of $89.3 \%$ (95\%, C.I. 86.6 to 91.6) (figure 1).

In recent years several studies (Brigtsen et al., 2015; Sheppard et al., 2016) have presented molecular based methods for typing of GBS isolates, and a standard GBS PCR method has been described (Imperi et al., 2010). The most recent molecular GBS typing method described is the Whole-Genome Sequencing, which has the advantage, that besides providing information on the capsular genes, it also can provide information on multilocus sequence type, analyses of relatedness to other sequenced isolates, and detailed phylogenetic analyses (Sheppard et al., 2016).

Our PCR assay provided a genotype for all the 41 phenotypically NT isolates (table 2). According to other studies, nearly $100 \%$ of GBS isolates can be genotyped by the use of molecular based methods (Brigtsen et al., 2015; Yao et al., 2013; Sheppard et al., 2016). This typing rate is much higher than the approximately $90 \%$ rate obtained by phenotypical assays (Brigtsen et al., 2015) (table 1).

The two GBS vaccines currently under Phase 2 trials are based on capsular polysaccharide conjugated vaccines, while another vaccine based on GBS surface proteins is under Phase 1 trial 
(Heath, 2016). The capsular polysaccharide based vaccines cover either serotype III or serotype Ia, Ib and IIII (Heart, 2016). Evaluating the predicted vaccine coverage for Danish invasive GBS isolates from 2010 and 2011 in this study, we found that genotyping suggested an apparent increase in predicted vaccine coverage of $6.9 \%$ in 2010 and $4.4 \%$ in 2011 . However, as these isolates were only typeable by molecular methods, the type identification represents lack of phenotypical expression and therefore possible lack of vaccine coverage.

In conclusion, in this study we found that the latex test and the LP test showed similar identification percentages. Because of the greater workload with LP test, we recommend this method to be used only for latex test NT isolates (figure 1).

Molecular typing methods are advantageous for the surveillance of GBS infections in terms of evaluating transmission chains and possible description of, e.g. early onset neonatal infections (Bergseng et al., 2009). In contrast, phenotypical methods must be applied when evaluating possible vaccine coverage or vaccine failures as well as planning of future capsular polysaccharide vaccines. Therefore, appropriate typing methods must be chosen according to the purpose of surveillance. Based on our findings, we suggest that general surveillance can be performed either by using the phenotypical procedure shown in figure 1 or by molecular techniques such as those described by Sheppard et al. (2016), depending on the laboratory capacity and cost. Coverage studies to provide data for possible future polysaccharide based GBS vaccines will require a phenotypical procedure. In case a polysaccharide GBS vaccine will be implemented in the future, phenotypical procedures will be necessary when evaluating vaccine failure in patients with infections caused by vaccine serotypes. Molecular techniques including MLST are necessary if information on clonal relation is needed, e.g. for identification of transmission chains in case of outbreaks or clustered infections. 
230

231

232

233

234

235

236

237

\section{Acknowledgements}

Kirsten Burmeister and Monja Hammer are acknowledged for their skilful laboratory work and input to this study. We acknowledge the Danish departments of clinical microbiology for submitting streptococcus group B isolates for national surveillance throughout the study period.

\section{REFERENCES}

1. Afshar B, Broughton K, Creti R, Decheva A, Hufnagel M, Kriz P, Lambertsen L, Lovgren M, Melin P, Orefici G, Poyart C, Radtke A, Rodriguez-Granger J, Sørensen UB, Telford J, Valinsky L, Zachariadou L; Members of the DEVANI Study Group, Efstratiou A. 2011. International external quality assurance for laboratory identification and typing of Streptococcus agalactiae (Group B streptococci). J Clin Microbiol 49:1475-82. doi: 10.1128/JCM.02365-10.

2. Ballard MS, Schønheyder HC, Knudsen JD, Lyytikäinen O, Dryden M, Kennedy KJ, Valiquette L, Pinholt M, Jacobsson G, Laupland KB; International Bacteremia Surveillance Collaborative. 2016. The changing epidemiology of group B Streptococcus bloodstream infection: a multi-national population-based assessment. Infect Dis (Lond) 48:386-91. doi: 10.3109/23744235.2015.1131330.

3. Bergseng H, Afset JE, Radtke A, Loeseth K, Lyng RV, Rygg M, Bergh K. 2009. Molecular and phenotypic characterization of invasive group B Streptococcus strains from infants in Norway 2006-2007. Clin Microbiol Infect 15:1182-5. doi:10.1111/j.14690691.2009.02789.x. 
4. Brigtsen AK, Dedi L, Melby KK, Holberg-Petersen M, Radtke A, Lyng RV, Andresen LL, Jacobsen AF, Fugelseth D, Whitelaw A. 2015. Comparison of PCR and serotyping of Group B Streptococcus in pregnant women: the Oslo GBS-study. J Microbiol Methods 108:31-5. doi: 10.1016/j.mimet.2014.11.001.

5. Bulkowstein S, Ben-Shimol S, Givon-Lavi N, Melamed R, Shany E, Greenberg D. 2016. Comparison of early onset sepsis and community-acquired late onset sepsis in infants less than 3 months of age. BMC Pediatr 16:82. doi:10.1186/s12887-016-0618-6.

6. Heath PT. 2016. Status of vaccine research and development of vaccines for GBS. Vaccine 34:2876-9. doi: 10.1016/j.vaccine.2015.12.072.

7. Imperi M, Pataracchia M, Alfarone G, Baldassarri L, Orefici G, Creti R. 2010. A multiplex PCR assay for the direct identification of the capsular type (Ia to IX) of Streptococcus agalactiae. J Microbiol Methods 80:212-4.

8. Lambertsen L, Ekelund K, Skovsted IC, Liboriussen A, Slotved HC. 2010. Characterisation of invasive group B streptococci from adults in Denmark 1999 to 2004. Eur J Clin Microbiol Infect Dis 29:1071-7. doi:10.1007/s10096-010-0941-z.

9. Le Doare K, Heath PT. 2013. An overview of global GBS epidemiology. Vaccine 31 Suppl 4:D7-12. doi:10.1016/j.vaccine.2013.01.009. Review.

10. Poyart C, Tazi A, Réglier-Poupet H, Billoët A, Tavares N, Raymond J, and Trieu-Cuot P. 2007. Multiplex PCR Assay for Rapid and Accurate Capsular Typing of Group B Streptococci. J Clin Microbiol 45:1985-1988.

11. Schrag SJ, Verani JR. 2013. Intrapartum antibiotic prophylaxis for the prevention of perinatal group B streptococcal disease: experience in the United States and implications for a potential group B streptococcal vaccine. Vaccine 31:Suppl 4:D20-6. doi: 10.1016/j.vaccine.2012.11.056. 
12. Sheppard AE, Vaughan A, Jones N, Turner P, Turner C, Efstratiou A, Patel D; Modernising Medical Microbiology Informatics Group, Walker AS, Berkley JA, Crook DW, Seale AC. 2016. Capsular Typing Method for Streptococcus agalactiae Using Whole-Genome Sequence Data. J Clin Microbiol 54:1388-90. doi:10.1128/JCM.0314215.

13. Slotved HC, Sauer S, Konradsen HB. 2002. False-negative results in typing of group B streptococci by the standard lancefield antigen extraction method. J Clin Microbiol 40:1882-3.

14. Slotved HC, Elliott J, Thompson T, Konradsen HB. 2003. Latex assay for serotyping of group B Streptococcus isolates. J Clin Microbiol 41:4445-7.

15. Slotved HC, Kong F, Lambertsen L, Sauer S, Gilbert GL. 2007. Serotype IX, a Proposed New Streptococcus agalactiae Serotype. J Clin Microbiol 45:2929-36.

16. Vinnemeier CD, Brust P, Owusu-Dabo E, Sarpong N, Sarfo EY, Bio Y, Rolling T, Dekker D, Adu-Sarkodie Y, Eberhardt KA, May J, Cramer JP. 2015. Group B Streptococci serotype distribution in pregnant women in Ghana: assessment of potential coverage through future vaccines. Trop Med Int Health 20:1516-1524.doi: 10.1111/tmi.12589.

17. Yao K, Poulsen K, Maione D, Rinaudo CD, Baldassarri L, Telford JL, Sørensen UB; Members of the DEVANI Study Group, Kilian M. 2013. Capsular gene typing of Streptococcus agalactiae compared to serotyping by latex agglutination. J Clin Microbiol 51:503-7. doi: 0.1128/JCM.02417-12. 


\section{Figure 1 (on next page)}

A description of the GBS phenotypical serotype identification procedure at Statens Serum Institut. 


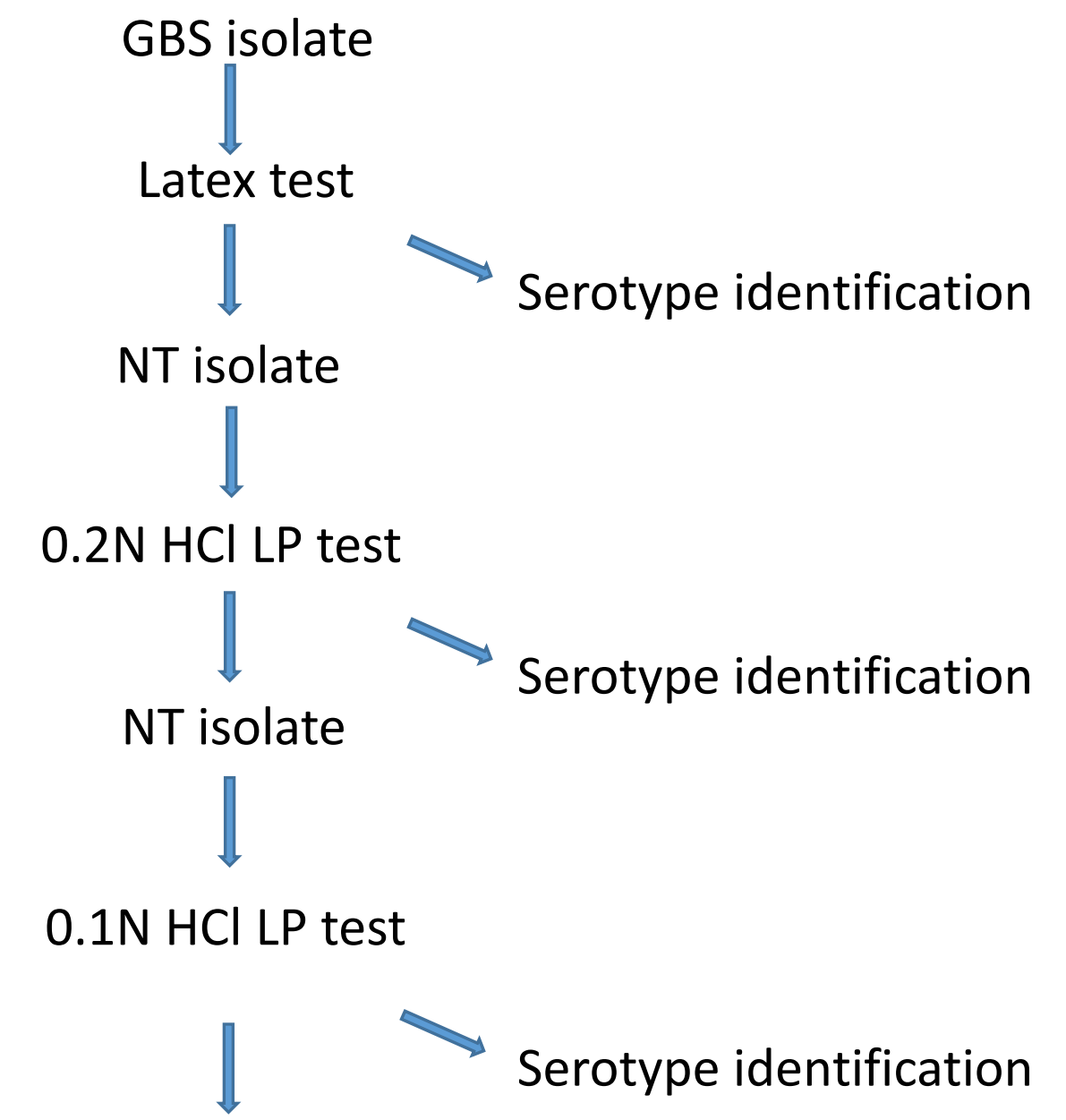

Isolate defined as phenotypical NT isolate 


\section{Table $\mathbf{1}$ (on next page)}

Comparison of Lancefield precipitation test ( $\mathrm{LP}$ test) $(0.2 \mathrm{~N} \mathrm{HCl}+0.1 \mathrm{~N} \mathrm{HCl})$ and latex test. 
1 Table 1. Comparison of Lancefield precipitation test (LP test) $(0.2 \mathrm{~N} \mathrm{HCl}+0.1 \mathrm{~N} \mathrm{HCl})$ and latex test.

\begin{tabular}{|c|c|c|c|c|c|c|c|c|c|c|c|c|}
\hline & \multicolumn{12}{|c|}{ Serotype assigned by LP test* } \\
\hline Serotype assigned by latex test** & Ia & $\mathrm{Ib}$ & II & III & IV & $\mathrm{V}$ & VI & VII & VIII & IX & NT & Total number \\
\hline Ia & 105 & & & & & & & & & & 1 & 106 \\
\hline $\mathrm{Ib}$ & & 49 & & & & & & & & & & 49 \\
\hline II & & & 57 & & & & & & & & 1 & 58 \\
\hline III & & & & 172 & & & & & & & & 172 \\
\hline IV & & & & & 24 & & & & & & & 24 \\
\hline $\mathrm{V}$ & & & & & & 73 & & & & & 6 & 79 \\
\hline VI & & & & & & & 4 & & & & 1 & 5 \\
\hline VII & & & & & & & & 1 & & & 1 & 2 \\
\hline VIII & & & & & & & & & 15 & & & 15 \\
\hline IX & & & & & & & & & & 5 & 1 & 6 \\
\hline NT & 8 & 4 & 3 & 13 & 2 & 4 & & & & & 66 & 100 \\
\hline $\begin{array}{c}\text { Total number } \\
(0.2 \mathrm{~N} \mathrm{HCl}+0.1 \mathrm{~N} \mathrm{HCl})\end{array}$ & $\begin{array}{c}113 \\
(112+1)\end{array}$ & $\begin{array}{c}53 \\
(53+0)\end{array}$ & $\begin{array}{c}60 \\
(60+0)\end{array}$ & $\begin{array}{c}185 \\
(182+3)\end{array}$ & $\begin{array}{c}26 \\
(26+0)\end{array}$ & $\begin{array}{c}77 \\
(76+1)\end{array}$ & $\begin{array}{c}4 \\
(4+0)\end{array}$ & $\begin{array}{c}1 \\
(1+0)\end{array}$ & $\begin{array}{c}15 \\
(12+3)\end{array}$ & $\begin{array}{c}5 \\
(4+1)\end{array}$ & $\begin{array}{c}77 \\
(77+0)\end{array}$ & $\begin{array}{c}616 \\
(607+9)\end{array}$ \\
\hline
\end{tabular}

2 *If Ia to IX $0.2 \mathrm{~N} \mathrm{HCl}$ was negative, then automatically Ia to IX $0.1 \mathrm{~N} \mathrm{HCl}$ was tested.

$3 * *$ If cross-reactions were observed with the Latex test, the isolate was considered to be non-typeable. 


\section{Table 2 (on next page)}

Results of genotyping of 39 phenotypically non-typable GBS isolates in 2010 and 2011 
1 Table 2. Results of genotyping of 39 phenotypically non-typable GBS isolates in 2010 and 2011

\begin{tabular}{|c|c|c|c|c|c|c|c|c|c|c|c|c|c|}
\hline & \multicolumn{9}{|c|}{ Genotype assigned by PCR } \\
\hline $\begin{array}{c}\text { Serotype assigned by Lancefield } \\
\text { Precipitation test* }\end{array}$ & Ia & Ib & II & III & IV & V & VI & VII & VIII & IX & NT & Total number of genotyped NT isolates & No PCR \\
\hline 2010 (143/173 isolates tested) & 4 & 1 & 1 & 7 & & 10 & & & 1 & & & 24 of total 30 \\
\hline 2011 (120/136 isolates tested) & 1 & 3 & 3 & 2 & & 4 & & & 1 & 1 & & 5 \\
\hline $\begin{array}{c}\text { Total number of isolates of each } \\
\text { genotype }\end{array}$ & 5 & 4 & 4 & 9 & 0 & 14 & 0 & 0 & 2 & 1 & 0 & 15 of total 16 \\
\hline
\end{tabular}

*Phenotypical identification excluding NT / total number of isolates)

3 If Ia to IX $0.2 \mathrm{~N} \mathrm{HCl}$ was negative, then automatically Ia to IX $0.1 \mathrm{~N} \mathrm{HCl}$ was tested.

4 


\section{Table 3 (on next page)}

Comparison of Lancefield Precipitation test (LP test) results with the PCR for 66 isolates. 
1 Table 3. Comparison of Lancefield Precipitation test (LP test) results with the PCR for 66

2 isolates.

\begin{tabular}{|c|c|c|c|c|c|c|c|c|c|c|c|c|}
\hline & \multicolumn{9}{|c|}{ Genotype assigned by PCR } \\
\hline $\begin{array}{c}\text { Serotype assigned by } \\
\text { LP-test }\end{array}$ & Ia & Ib & II & III & IV & V & VI & VII & VIII & IX & NT & Total number \\
\hline Ia & 4 & & & & & & & & & & & 4 \\
\hline Ib & & 2 & & & & & & & & & & 2 \\
\hline II & & & 2 & & & & & & & & & 2 \\
\hline III & & & 2 & 7 & & & & & & & & 9 \\
\hline IV & & & & & 2 & & & & & & & 2 \\
\hline V & & & & & & & 1 & & & & & \\
\hline VI & & & & & & & & & & & & \\
\hline VII & & & & & & & & & & & & 0 \\
\hline VIII & 5 & 5 & 4 & 9 & & 15 & & & 2 & 1 & & 0 \\
\hline IX & 9 & 7 & 8 & 16 & 2 & 20 & 1 & 0 & 2 & 1 & 0 & 41 \\
\hline NT & & & & & & & & & & \\
\hline Total number & & & & & & & & & & & \\
\hline
\end{tabular}

3 *If no reaction with Ia to IX was found with $0.2 \mathrm{~N} \mathrm{HCl}$, then Ia to IX were tested with $0.1 \mathrm{~N} \mathrm{HCl.}$

4 Red numbers represent isolates that were identified with contradicting typing. 
Table 4 (on next page)

Comparison of Latex test result with the PCR. 
1

2 Table 4. Comparison of Latex test result with the PCR.

\begin{tabular}{|c|c|c|c|c|c|c|c|c|c|c|c|c|}
\hline & \multicolumn{12}{|c|}{ Genotype assigned by PCR } \\
\hline $\begin{array}{c}\text { Serotype assigned by } \\
\text { Latex test }\end{array}$ & Ia & $\mathrm{Ib}$ & $\overline{\text { II }}$ & III & IV & $\mathrm{V}$ & VI & VII & VIII & IX & NT & Total number \\
\hline $\mathrm{Ia}$ & 4 & & & & & & & & & & & 4 \\
\hline $\mathrm{Ib}$ & & & & & & & & & & & & 0 \\
\hline II & & & & & & & & & & & & 0 \\
\hline III & & & & 3 & & & & & & & & 3 \\
\hline IV & & & & & 1 & & & & & & & 1 \\
\hline $\mathrm{V}$ & & & & & & 6 & & & & & & 6 \\
\hline $\mathrm{VI}$ & & & & & & 1 & 1 & & & & & 2 \\
\hline VII & & & & & & 1 & & & & & & 1 \\
\hline VIII & & & & & & & & & & & & 0 \\
\hline IX & & & & & & & & & & & & 0 \\
\hline NT & 5 & 7 & 8 & 13 & 1 & 12 & & & 2 & 1 & & 49 \\
\hline Total number & 9 & 7 & 8 & 16 & 2 & 20 & 1 & 0 & 2 & 1 & 0 & 66 \\
\hline
\end{tabular}

3 *If cross-reactions were observed with the Latex test, the isolate was considered to be non-

4 typeable.

5 Red numbers represent two isolates that were non-typeable with $0.2 \mathrm{~N} \mathrm{HCl}$ and $0.1 \mathrm{~N} \mathrm{HCl}$.

6 